Article

\title{
LoRa 2.4 GHz Communication Link and Range
}

\author{
Thomas Janssen $\mathbb{D}^{D}$, Noori BniLam ${ }^{\mathbb{D}}$, Michiel Aernouts ${ }^{\mathbb{D}}$, Rafael Berkvens ${ }^{\mathbb{D}}$ \\ and Maarten Weyn * (D)
}

IDLab-Faculty of Applied Engineering, University of Antwerp-imec, Sint-Pietersvliet 7, 2000 Antwerp, Belgium; thomas.janssen@uantwerpen.be (T.J.); noori.bnilam@uantwerpen.be (N.B.);

michiel.aernouts@uantwerpen.be (M.A.); rafael.berkvens@uantwerpen.be (R.B.)

* Correspondence: maarten.weyn@uantwerpen.be

Received: 9 July 2020; Accepted: 4 August 2020; Published: 5 August 2020

check for updates

\begin{abstract}
Recently, Semtech has released a Long Range (LoRa) chipset which operates at the globally available $2.4 \mathrm{GHz}$ frequency band, on top of the existing sub-GHz, km-range offer, enabling hardware manufacturers to design region-independent chipsets. The SX1280 LoRa module promises an ultra-long communication range while withstanding heavy interference in this widely used band. In this paper, we first provide a mathematical description of the physical layer of LoRa in the $2.4 \mathrm{GHz}$ band. Secondly, we investigate the maximum communication range of this technology in three different scenarios. Free space, indoor and urban path loss models are used to simulate the propagation of the $2.4 \mathrm{GHz}$ LoRa modulated signal at different spreading factors and bandwidths. Additionally, we investigate the corresponding data rates. The results show a maximum range of $133 \mathrm{~km}$ in free space, $74 \mathrm{~m}$ in an indoor office-like environment and $443 \mathrm{~m}$ in an outdoor urban context. While a maximum data rate of $253.91 \mathrm{kbit} / \mathrm{s}$ can be achieved, the data rate at the longest possible range in every scenario equals $0.595 \mathrm{kbit} / \mathrm{s}$. Due to the configurable bandwidth and lower data rates, LoRa outperforms other technologies in the $2.4 \mathrm{GHz}$ band in terms of communication range. In addition, both communication and localization applications deployed in private LoRa networks can benefit from the increased bandwidth and localization accuracy of this system when compared to public sub-GHz networks.
\end{abstract}

Keywords: low power wide area networks; LPWAN; LoRaWAN; LoRa; $2.4 \mathrm{GHz}$; wireless sensor networks; path loss modeling; range estimation

\section{Introduction}

More than a decade ago, Long Range (LoRa) was invented to-as the name indicates-provide a low power wide area network (LPWAN) protocol operating at sub-GHz frequencies. Because of local spectrum regulations, LoRa hardware modules need to be adapted to operate in different frequency bands. For example, in the US, the $915 \mathrm{MHz}$ band is used, while in Europe, most LoRa chipsets operate in the $868 \mathrm{MHz}$ band. In the past, we have conducted extensive research on communication and localization with sub-GHz LPWAN.

Semtech, the founding member of the LoRa Alliance, recently released a LoRa chipset operating at $2.4 \mathrm{GHz}$. The move from sub-GHz to $2.4 \mathrm{GHz}$ was mainly done in order to use the globally available 2.4 GHz Industrial, Scientific and Medical (ISM) band. This tackles the problem of having to develop multiple chipsets which operate at different frequency bands, thus paving the way for the development of a universal chipset which can operate anywhere in the world. This is especially valid for track and trace applications, where goods cross different zones worldwide. Nevertheless, the potential benefits of LoRa in the $2.4 \mathrm{GHz}$ band have not yet been investigated thoroughly, which constitutes the main objective of this paper. 
In general, the range of sub-GHz LPWAN varies from a few kilometers in urban environments to more than $10 \mathrm{~km}$ in rural environments. Obviously, the maximum communication range between the transmitter and receiver also depends on the used frequency band. The goal of this research is to study the inverse relationship between the maximum communication range and the corresponding data rate of LoRa in the $2.4 \mathrm{GHz}$ band.

Since sub-GHz LoRa devices are very limited in terms of their number of possible transmissions-i.e., to comply with duty cycle regulations-they are typically used for applications that do not require frequent communication. For example, Sendra et al. proposed a LoRa-based forest fire detection system that transmits sensor measurements every $28 \mathrm{~min}$ [1]. Besides this, the long-range communication benefit of sub-GHz LoRa has been exploited to provide emergency services in GPS-less environments [2]. Other application examples with sub-GHz LoRa include smart meter reading, environmental monitoring, smart farming and smart building applications [3,4]. On the other hand, LoRa devices that operate at $2.4 \mathrm{GHz}$ are able to transmit at higher data rates because of the higher available bandwidth [5]. Consequently, this technology can offer a balance for applications that require a higher data rate than LPWANs and a longer communication range than classic $2.4 \mathrm{GHz}$ technologies such as Wi-Fi and Bluetooth. Additionally, a higher bandwidth also allows for more accurate time-based localization [6]. Thus, LoRa at $2.4 \mathrm{GHz}$ represents an interesting solution for a variety of applications that involve indoor localization, such as warehouse management, but also for applications that require outdoor localization, such as construction site monitoring, livestock tracking, etc. Moreover, the adoption of this technology can add flexibility to applications that require consistent asset tracking in both indoor and harsh outdoor environments; e.g., smart ports.

The main contributions of this work are the following:

- We provide an overview of the physical layer of LoRa operating at $2.4 \mathrm{GHz}$.

- We discuss the maximum communication range and data rate in three different scenarios: free space, indoor and urban environments.

- We discuss the impact of moving from LoRa at sub-GHz bands to $2.4 \mathrm{GHz}$ on communication and localization applications.

The remainder of this paper is structured as follows. Section 2 contains related work regarding LoRa in both sub-GHz bands and the $2.4 \mathrm{GHz}$ band. Subsequently, a mathematical background of LoRa at $2.4 \mathrm{GHz}$ is provided in Section 3. Next, three path loss models are presented in Section 4 in order to estimate the maximum communication range and corresponding data rate in a free space and in indoor and urban environments. The results of these estimations are shown in Section 5 and compared to other technologies operating in the $2.4 \mathrm{GHz}$ band in Section 6, which also discusses the impact on the application potential of LoRa at $2.4 \mathrm{GHz}$. Finally, general conclusions are drawn in Section 7.

\section{Related Work}

With the aim of providing a low power, long-range wireless communication protocol, Semtech acquired the startup company Cycleo, which developed LoRa in 2009. The authors in [7] provided an in-depth analysis of the functional components of both the physical (LoRa) and data link (LoRaWAN) layer of the popular LPWAN protocol operating in unlicensed bands.

Sub-GHz LoRa-based communication has been studied thoroughly in the past decade. Acting as a layer above LoRa, LoRaWAN is widely used to communicate small messages over large areas. A LoRaWAN network can fully cover a city-scale environment with only a few gateways. The mobility of LoRaWAN has been addressed in [8]. The forwarding of an uplink message by multiple gateways ensures that a handover can take place without any loss of data. In another study, a robust frame detection algorithm was proposed in order to detect LoRa-modulated frames with minimal complexity implementations [9]. Furthermore, a frame relay strategy was found to be a feasible way to improve the link quality of poorly connected nodes and successfully extend the range of LoRaWAN [10]. 
In addition to the communication aspect of LPWAN, a significant amount of research, both from academic and industrial perspectives, has involved the location objects with of sub-GHz LPWAN. We conducted extensive research on localization with different LPWAN technologies. We evaluated the accuracy of received signal strength (RSS)-based localization algorithms for Sigfox [11], LoRaWAN [12] and NB-IoT [13]. We also provided an angle of arrival (AoA) estimation solution that is suitable for use in sub-GHz bands [14,15]. Furthermore, we devised a probabilistic localization model that combines time difference of arrival (TDOA) and AoA estimations in a sub-GHz LoRa network [6].

Recently, Semtech released the SX1280 chipset, which operates at $2.4 \mathrm{GHz}$ [5]. In an application note, the company provides an introduction to time-of-flight (ToF) ranging possibilities with the radio chip [16]. A proof-of-concept implementation for coherent multi-channel ranging with this LoRa radio chip is provided in [17].

A few concerns are raised by the move from sub-GHz frequencies to the widely used unlicensed 2.4 GHz ISM band. The interference properties of wireless local area networks (WLAN, also denoted as Wi-Fi) devices operating in this band were characterized in [18]. In another study, coexistence issues with Wi-Fi and LoRa 2.4 GHz were addressed [19]. The theoretical assumptions about the high robustness of the system against interference are confirmed. However, it was found that the robustness highly depends on both the configuration of LoRa and the properties of interfering technologies. Moreover, Polak et al. used different bandwidths in their evaluation and compared them to the bandwidths that are currently available for LoRa at $2.4 \mathrm{GHz}$.

The maximum communication range in the $2.4 \mathrm{GHz}$ band strongly depends on the technology and its respective transmission power. For the latest Bluetooth Low Energy (BLE 5) standard, the maximum communication ranges in different scenarios are summarized in [20]. Typical ranges are $50 \mathrm{~m}$ in an indoor scenario, $165 \mathrm{~m}$ in an outdoor non-line-of-sight (NLoS) scenario and $780 \mathrm{~m}$ in a LoS scenario. In this research, we will investigate the range of $2.4 \mathrm{GHz}$ LoRa in a free space and in indoor and outdoor scenarios.

\section{LoRa in the $2.4 \mathrm{GHz}$ Band}

The physical layer of LoRa is a proprietary and closed source. Therefore, there are no official references or protocol specifications for the transmitted RF signal [21]. Accordingly, several research groups have been working to provide an understanding of the LoRa modulation scheme in the sub-GHz frequency band. Vangelista [22], for instance, has provided a mathematical model, called Frequency Shift Chirp Modulation (FSCM), that describes the LoRa modulation process. The same model has been adopted by Bernier et al. [9]. On the other hand, Knight [21], Robyns et al. [23] and Ghanaatian et al. [24] have provided a model called Chirp Spread Spectrum (CSS) modulation based on the reverse engineering of LoRa's physical layer. Even though the formulation of the LoRa modulation scheme in the literature has been provided for the sub-GHz frequency band, the basic response of the modulation is expected to be the same for LoRa signals in the $2.4 \mathrm{GHz}$ frequency band. Therefore, in this section, we modify the available physical layer models of sub-GHz LoRa to make them suitable for use in the $2.4 \mathrm{GHz}$ frequency band.

Assume $x_{s}(k)$ is the transmitted LoRa sample; then, the received sampled signal $x_{r}(k)$ with index $k$ can be expressed as

$$
x_{r}(k)=a_{r} x_{s}(k-\tau) e^{i 2 \pi \Delta f k}+\omega(k),
$$

where $a_{r}<1$ is the received signal amplitude, $\tau$ is the time delay of the sample $x_{s}(k)$ at the receiver, $\Delta f$ is the frequency offset between the transmitter and the receiver, and $\omega(k)$ is the identically independently distributed (i.i.d.) complex-valued Gaussian noise with zero-mean and variance $\sigma^{2}$; i.e., $C N\left(0, \sigma^{2}\right)$. The time and frequency synchronization are beyond the scope of this paper. Therefore, in the following, we will consider a simplified version of (1), shown in (2).

$$
x_{r}(k)=a_{r} x_{s}(k)+\omega(k)
$$


The LoRa standard linear upchirp—also called a base chirp—can be expressed as [23,24]

$$
x_{S}(k)=e^{i 2 \pi\left(\frac{\mathrm{BW}}{2 K} k^{2}+f_{\mathrm{o}} k\right)},
$$

where BW is the operational bandwidth of the LoRa signal in the $2.4 \mathrm{GHz}$ frequency band (as shown in Table 1) and $K=2^{\mathrm{SF}} / \mathrm{BW}$ is the symbol duration, with SF representing the spreading factor (also shown in Table 1). Finally, $f_{\circ}$ is the initial frequency, which can be expressed as

$$
f_{\circ}=s \frac{\mathrm{BW}}{2^{\mathrm{SF}}},
$$

where $s \in\left\{0,1 \ldots 2^{\mathrm{SF}}\right\}$ is the transmitted data symbol. Setting $s=0$ results in an upchirp, in which the frequency continuously increases during the symbol duration $K$.

We can also present (3) as

$$
x_{s}(k)=W_{K}^{\frac{\mathrm{BW}}{2} k^{2}+K f_{\circ} k}, \quad W_{K}=e^{i 2 \pi / K} .
$$

Table 1. Parameters used for path loss modeling.

\begin{tabular}{llll}
\hline Model Parameter & Symbol & Value & Unit \\
\hline Frequency & $f$ & 2.4 & $\mathrm{GHz}$ \\
Spreading factor & $S F$ & $5-12$ & - \\
Bandwidth & $B W$ & $203 / 406 / 812 / 1625$ & $\mathrm{kHz}$ \\
Code rate & $R_{C}$ & $4 / 5$ & - \\
Transmission power & $P_{T X}$ & 12.5 & $\mathrm{dBm}$ \\
Transmitter antenna gain & $G_{T X}$ & 2 & $\mathrm{dBi}$ \\
Transmitter cable losses & $L_{T X}$ & 2 & $\mathrm{~dB}$ \\
Fading margin & $L_{m}$ & 0 & $\mathrm{~dB}$ \\
Receiver antenna gain & $G_{R X}$ & 2 & $\mathrm{dBi}$ \\
Receiver cable losses & $L_{R X}$ & 2 & $\mathrm{~dB}$ \\
Base station height & $h_{b}$ & 20 & $\mathrm{~m}$ \\
Mobile station height & $h_{m}$ & 2 & $\mathrm{~m}$ \\
\hline
\end{tabular}

The model in (5) is the linearly cyclically shifted version of a base Zadoff-Chu (ZC) sequence [25]. The ZC sequence possesses a unique autocorrelation property, in which the periodic autocorrelation is orthogonal (i.e., equal to zero) for all shifted replicas [26]. Therefore, the LoRa communication protocol uses this unique property to impose a random multiple access technique. Accordingly, an efficient utilization of the unlicensed spectrum can be obtained. The correlation between the received signal and the base chirp leads to

$$
\begin{aligned}
z(k) & =\frac{1}{K} \sum_{p=0}^{K-1} x(k+p) x_{s}^{*}(k)_{\bmod K} \\
& =\frac{1}{K} \sum_{p=0}^{K-1}\left(a_{r} x_{s}(k+p)+\omega(k+p)\right) x_{s}^{*}(k), \\
& =\left\{\begin{array}{cc}
a_{r} E_{s}+v_{\omega} & \text { for } p=0 \\
v_{\omega} & \text { for } p \neq 0
\end{array}\right.
\end{aligned}
$$

where $E_{s}$ is the energy of the symbol $x_{s}$. Furthermore, $v_{\omega}$ is the correlation between complex noise and the base chirp, which can be expressed as

$$
v_{\omega}=\frac{1}{K} \sum_{p=0}^{K-1} x_{s}^{*}(k) \omega(k+p),
$$


in which $v_{\omega} \sim C N\left(0, \sigma^{2} / K\right)$.

Figure 1 presents two received LoRa signals that constitute eight preamble (upchirp) symbols at $2.4 \mathrm{GHz}$ with a bandwidth equal to $812 \mathrm{kHz}$. The short signal was transmitted at an SF of 9, while the longer signal was transmitted at an SF of 10. Figure 1a,b shows the combined received signals in the time domain and in the spectrogram (i.e., time and frequency) domain, respectively. Figure 1c,d shows the cross-correlation functions (6) when the received signals have been cross-correlated with base chirps of the SF equal to 10 and 9, respectively. It is clear that the two signals can be distinguished correctly, even though they interfere with each other. The unique orthogonality property of the ZC sequence allows the LoRa communication system to provide a multiple access technique in the $2.4 \mathrm{GHz}$ frequency band.

a)

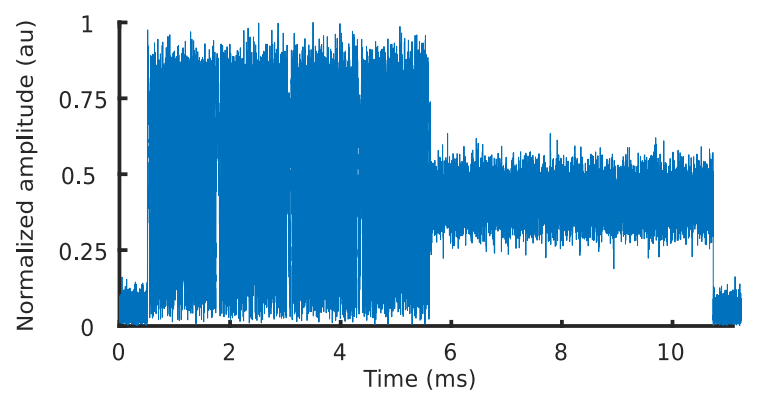

b)

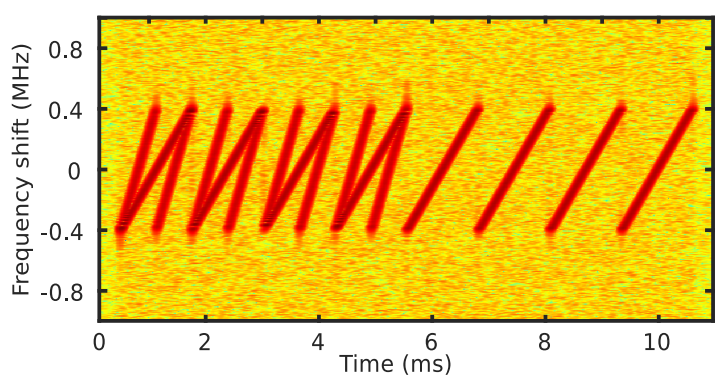

c)

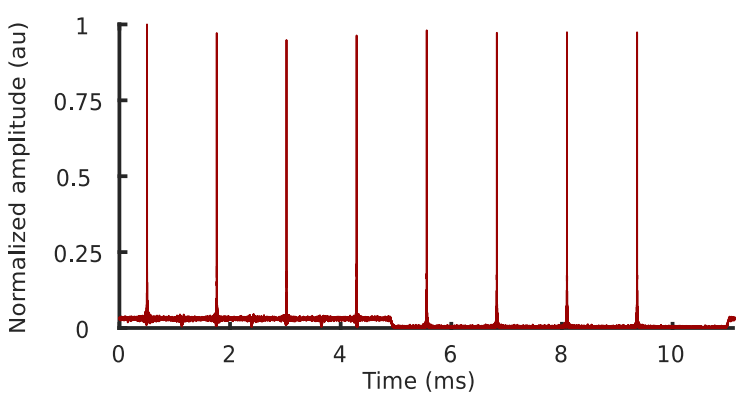

d)

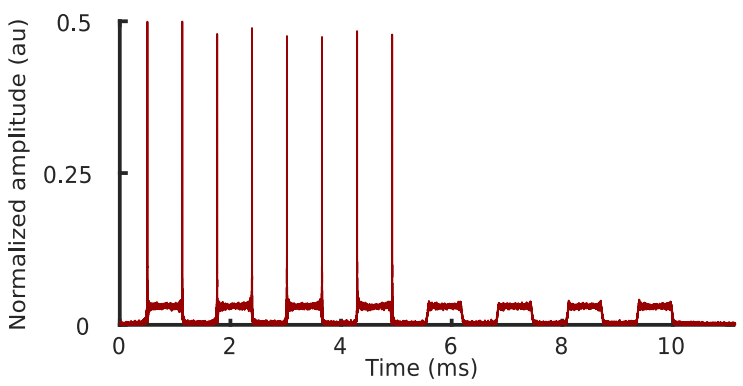

Figure 1. Two received Long Range (LoRa) signals constitute eight preamble upchirp symbols at $2.4 \mathrm{GHz}$ with a bandwidth equal to $812 \mathrm{kHz}$. The spreading factor (SF) of the short signal, which ended after approximately $5 \mathrm{~ms}$, is equal to 9 , while the $\mathrm{SF}$ of the long-duration signal is equal to 10 . Figures (a) and (b) represent the combined received signals in the time domain and in the spectrogram (i.e., time and frequency) domain, respectively. Figures (c) and (d) are the cross-correlation functions (6) when the received signals have been cross-correlated with base chirps of the SF equal to 10 and 9, respectively.

\section{Path Loss Modeling}

In order to obtain the maximum communication range of a LoRa signal at $2.4 \mathrm{GHz}$ (further denoted as $d$ ), we need to find the maximum link budget for which the signal can be received properly; i.e., at the receiver sensitivity $P_{R X}$. This receiver sensitivity depends on two key factors: the used spreading factor (SF) and bandwidth (BW). While the SF can range from 5 to 12 , the possible bandwidths of LoRa at $2.4 \mathrm{GHz}$ are 203, 406, 812 and $1625 \mathrm{kHz}$. Furthermore, the combination of a certain SF and BW results in a certain data rate, along with the receiver sensitivity, as shown in Table 2. The raw data rate $R_{b}$, expressed in $\mathrm{kbit} / \mathrm{s}$, can be calculated as

$$
R_{b}=\frac{S F * B W}{2^{S F}},
$$

with SF and BW as defined in Table 1. As an example, a LoRa signal transmitted with an SF of 8 and a $\mathrm{BW}$ of $406 \mathrm{kHz}$ results in a receiver sensitivity of $-116 \mathrm{dBm}$ and a data rate of $12.69 \mathrm{kbit} / \mathrm{s}$. The receiver 
sensitivities and data rates used in this work originate from the datasheet of the Semtech SX1280 LoRa module [5].

Table 2. Receiver sensitivities $\left(P_{R X}\right.$ in $\left.\mathrm{dBm}\right)$ and corresponding data rates $\left(R_{D}\right.$ in $\left.\mathrm{kbit} / \mathrm{s}\right)$ of the SX1280 LoRa module for every combination of spreading factors (SFs) and bandwidths (BWs).

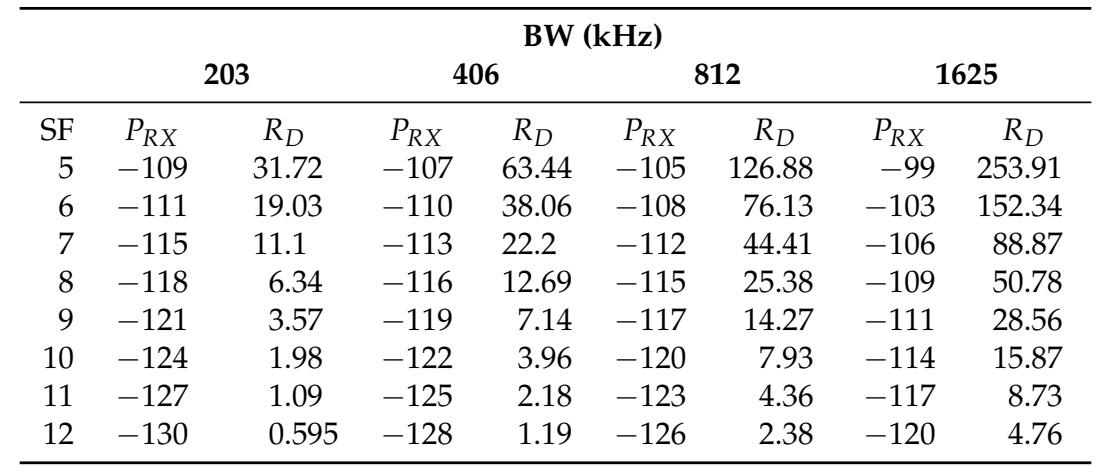

The total link budget of a wireless communication signal propagating from the transmitter to receiver can be represented as [27]

$$
P_{R X}=P_{T X}+G_{T X}-L_{T X}-L_{p}(d)+G_{R X}-L_{R X}
$$

where $P_{R X}$ is the received power in $\mathrm{dBm}, P_{T X}$ is the transmission power in $\mathrm{dBm}, G_{T X}$ is the antenna gain at the transmitter in $\mathrm{dBi}, L_{T X}$ is the cable loss in $\mathrm{dB}, L_{p}(d)$ is the path loss in $\mathrm{dB}$ in terms of the function from the distance $d, G_{R X}$ is the antenna gain at the receiver in $\mathrm{dB}$ and $L_{R X}$ is the cable loss at the receiver in $\mathrm{dB}$. Except for the path loss, all these parameters are set to typical values which are commonly used when simulating a wireless communication link between two dipole antennas [27]. The values of these parameters are summarized in Table 1.

The path loss $L_{p}(d)$ is defined as the propagation loss caused by the signal traveling from the transmitter to receiver over a distance $d$. The goal in this research is to maximize $d$ while still being able to successfully receive the LoRa-modulated signal at the receiver. For the sake of simplicity, the simulated LoRa signal contains eight preamble symbols and no payload bytes.

Depending on the environment, the path loss should be modeled differently. Therefore, in the next three subsections, we discuss indoor, outdoor (urban) and free space path loss models to translate the propagation loss into a distance between the transmitter and receiver. The parameters required by these models are also summarized in Table 1 . No fade margin is taken into account; this is further discussed in Section 6.

\subsection{Free Space Environment}

The first scenario can be described as a free space environment in which there is a line of sight (LoS, i.e., the primary Fresnel zone is to be at least $60 \%$ clear.) between the TX and RX locations. In this case, we can use the widely used Free Space Path Loss (FPSL) model to evaluate the maximum communication range. This model calculates the loss between two isotropic radiators in free space, without considering any obstacles, reflections or interference. The model solely relies on the frequency and distance between the transmitter and receiver to calculate the path loss:

$$
L_{p, L o S}(d)=32.44+20 \log _{10}(f)+20 \log _{10}(d)
$$

where $f$ is in MHz and $d$ is in $\mathrm{km}$. The combination of a given SF and BW yields a certain sensitivity $P_{R X}$. Consequently, given the maximum path loss obtained from (9), we can calculate the maximum distance as

$$
d=10^{\left(L_{p, L o S}(d)-32.44-20 \log _{10}(f)\right) / 20} .
$$




\subsection{Indoor Environment}

In the second scenario, we evaluate the maximum communication range in an indoor environment. To this end, we slightly adapt a heuristic algorithm that was developed based on real measurements in an office-like environment [28]. The path loss model is based on the Indoor Dominant Path (IDP) model, which focuses on the dominant path between the TX and RX location. In general, the total path loss is the sum of the distance loss, accumulated wall loss and interaction loss and can be calculated as

$$
L_{p, i n}(d)=L_{p_{0}}\left(d_{0}\right)+10 * n * \log _{10}\left(\frac{d}{d_{0}}\right)+\sum_{i} L_{W_{i}}+\sum_{j} L_{B_{j}},
$$

where $L_{p_{0}}\left(d_{0}\right)$ represents the path loss at a distance $d_{0}$ and $n$ is the path loss exponent. The accumulated wall loss is the sum of losses $L_{W_{i}}$ caused by each wall along the dominant path. Finally, the interaction loss is the sum of losses $L_{B_{j}}$ caused by all directional changes of the propagating signal.

Given the semi-empirical nature of this path loss model, some parameters need to be set to commonly used values in order to provide a generally applicable model that can predict ranges in other indoor environments. Therefore, $L_{p_{0}}\left(d_{0}\right)$ is set to $40 \mathrm{~dB}$ at a distance $d_{0}=1 \mathrm{~m}$, as suggested in [28]. The path loss exponent is set to $n=5$, which is generally used for obstructed paths inside buildings [29]. For the accumulated wall and interaction loss, values of 6 and 3 dB have been taken into account, as found specifically for the office-like environment in [28]. Consequently, the path loss model can be simplified to

$$
L_{p, i n}(d)=40+5 * 10 * \log _{10}(d)+6+3
$$

Thus, the range can be empirically estimated based on the path loss:

$$
d=10^{\left(L_{p, i n}(d)-49\right) / 50} .
$$

\subsection{Urban Environment}

An urban path loss model is used in the third scenario to evaluate the range of LoRa at $2.4 \mathrm{GHz}$ in an outdoor city-scale environment. The Okumura-Hata Urban Path Loss model is an empirical model that is often used in sub-GHz wireless communication systems. While the COST-231 urban model extended its use up to $2 \mathrm{GHz}$, the Electronic Communication Committee (ECC) modified the original Okumura-Hata model to work with frequencies up to (and beyond) $3 \mathrm{GHz}$ in the ECC-33 model [30,31]. Therefore, the ECC-33 model is suitable to evaluate the maximum communication range of LoRa at $2.4 \mathrm{GHz}$ in an urban environment. The path loss equation for this model is given by

$$
L_{p, u r b a n}(d)=A_{f s}+A_{b m}+G_{b}+G_{m}
$$

where $A_{f s}$ is the free space attenuation, $A_{b m}$ is the basic median path loss, $G_{b}$ is the base station height gain factor and $G_{r}$ is the receiver height gain factor, which can be calculated as

$$
\begin{aligned}
A_{f s}= & 92.4+20 \log _{10}(d)+20 \log _{10}(f), \\
A_{b m}= & 20.41+9.83 \log _{10}(d)+7.894 \log _{10}(f) \\
& +9.56\left[\log _{10}(f)\right]^{2}, \\
G_{b}= & \log _{10}\left(\frac{h_{b}}{200}\right)\left\{13.958+5.8\left[\log _{10}(d)\right]^{2}\right\}, \text { and } \\
G_{m}= & {\left[42.57+13.7 \log _{10} f\right]\left[\log _{10}\left(h_{m}\right)-0.585\right] }
\end{aligned}
$$

for medium-sized urban environments. Given the complexity of this set of equations, we extract the maximum range by iterating over values of $d$ from $1 \mathrm{~m}$ to $10 \mathrm{~km}$ and solving the optimization problem given a certain path loss $L_{p, u r b a n}(d)$. 


\section{Range Versus Data Rate: Results}

Figures 2-4 show the maximum communication range and corresponding data rate at each combination of SF and bandwidth for the free space and indoor and urban environments, respectively. In all cases, the highest possible data rate decreases in a logarithmic way when the communication range between the transmitter and receiver increases.

Using the Free Space Path Loss model, it is found that a $2.4 \mathrm{GHz}$ LoRa signal can travel up to $133 \mathrm{~km}$ in free space and still be received properly. Obviously, this is only a theoretical range and cannot be realized in real-world environments.

The performance of a more realistic indoor path loss model has been visualized in Figure 3. When transmitting with a spreading factor of 12 and the lowest bandwidth (i.e., $203 \mathrm{kHz}$ ), the path loss equals $142.5 \mathrm{~dB}$. Consequently, a maximum communication range of $74 \mathrm{~m}$ can be achieved. Furthermore, the highest possible data rate at that range becomes $0.595 \mathrm{kbit} / \mathrm{s}$. At the other extreme, the highest achievable data rate of $253.91 \mathrm{kbit} / \mathrm{s}$ is possible at a range of up to $18 \mathrm{~m}$.

Finally, the communication range of the urban ECC-33 path loss model varies from $3 \mathrm{~m}$ at the highest achievable data rate of $253.91 \mathrm{kbit} / \mathrm{s}$ to $443 \mathrm{~m}$ at the lowest possible data rate of $0.595 \mathrm{kbit} / \mathrm{s}$.

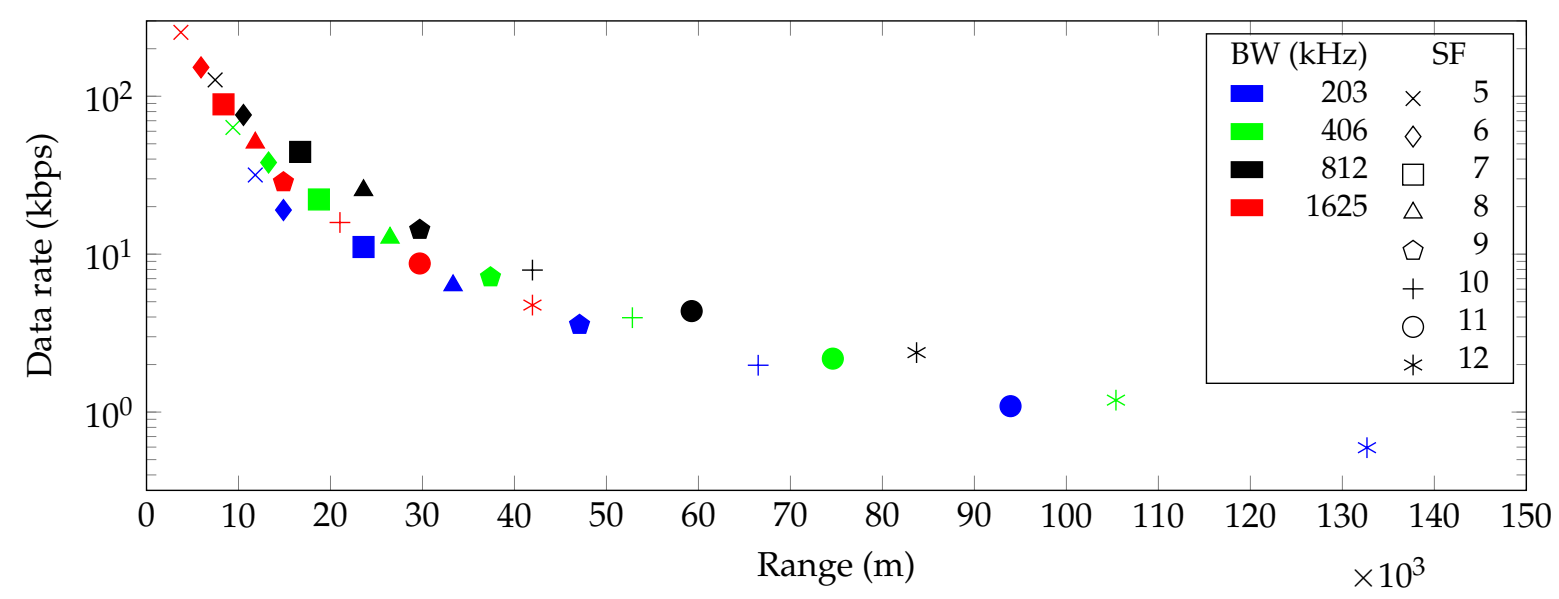

Figure 2. Communication range and data rate for every combination of spreading factor (SF) and bandwidth (BW) in a free space line of sight (LoS) environment.

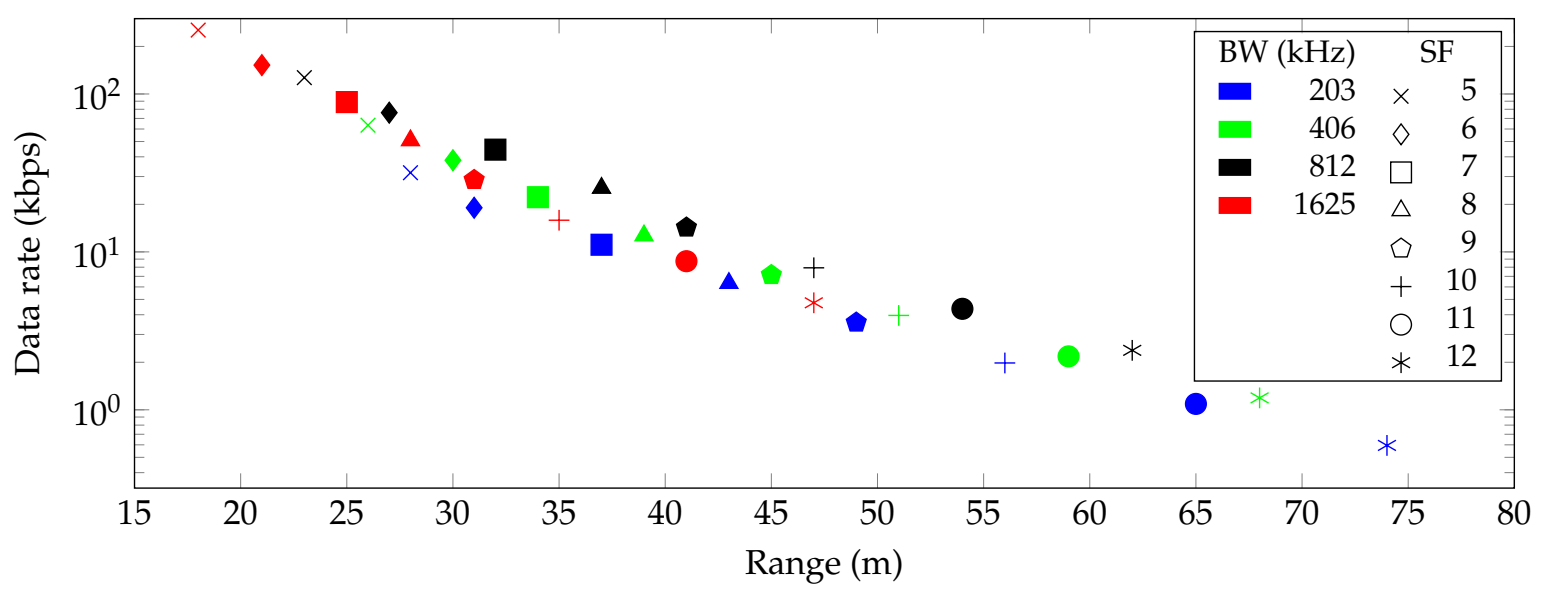

Figure 3. Communication range and data rate for every combination of spreading factor (SF) and bandwidth (BW) in an indoor environment. 


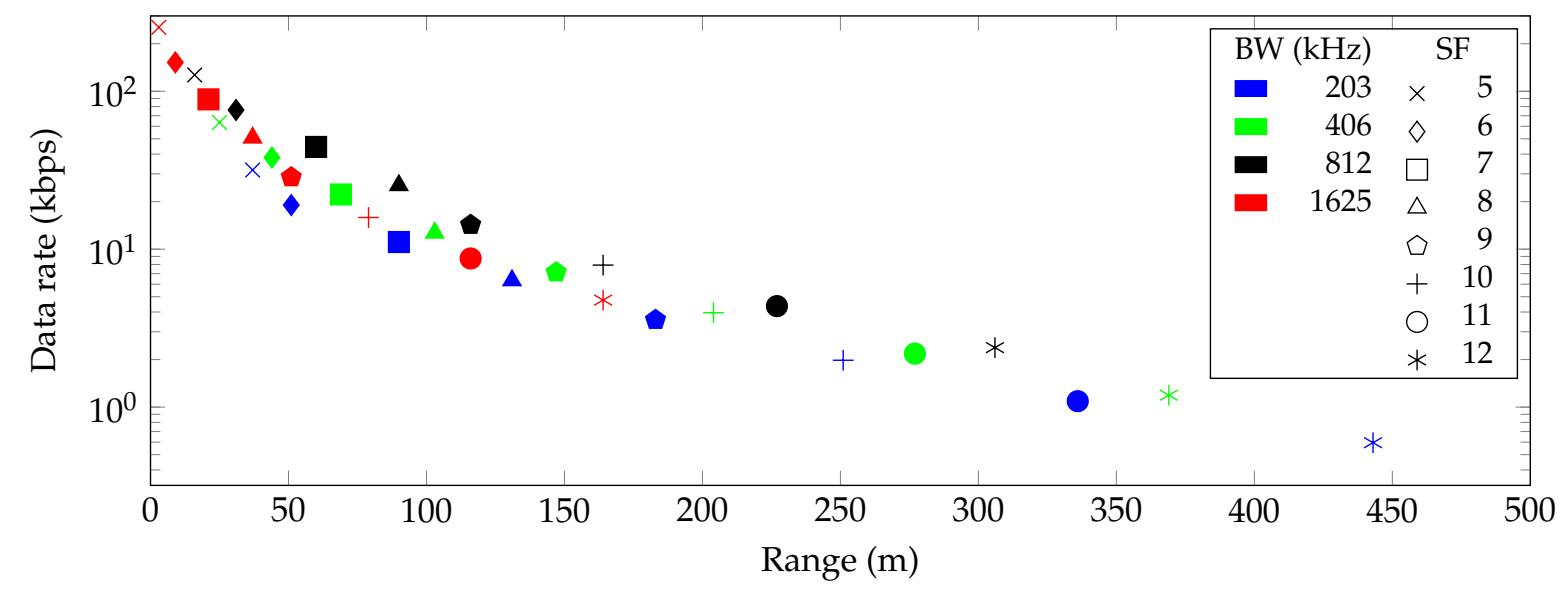

Figure 4. Communication range and data rate for every combination of spreading factor (SF) and bandwidth (BW) in an urban environment.

\section{Discussion}

We investigated the maximum communication range of LoRa in the $2.4 \mathrm{GHz}$ band, which is defined as the maximum distance between a transmitter and receiver at which a LoRa-modulated message can be received properly. Our investigations included three environments: LoS free space, NLoS indoor and urban outdoor. In all three scenarios, we maximized the range by reducing the bandwidth and increasing the spreading factor.

Based on the total link budget of the wireless communication system, including receiver sensitivity, antenna gains and cable losses, we were able to estimate the range of LoRa at $2.4 \mathrm{GHz}$. It is important to note that we did not include a fade margin in the link budget calculations. The fade margin can be defined as the level of received power in excess of that required for a specified minimum level of system performance. The reason for excluding this loss parameter in (9) is the high variability of fade margin in different scenarios. For instance, a $5 \mathrm{~dB}$ fade margin decreases the maximum urban range from $867 \mathrm{~m}$ to $576 \mathrm{~m}$, while a $10 \mathrm{~dB}$ fade margin further decreases the range to $164 \mathrm{~m}$. Thus, this should be taken into account when analyzing the results. Nonetheless, the largest factor by far in a link budget is the path loss.

The free space line-of-sight scenario resulted in a theoretical maximum range of $133 \mathrm{~km}$ when transmitting at the highest SF and using the lowest bandwidth. In reality, the signal will always have to cope with obstacles, multipath propagation effects and interference with other signals. Therefore, these ranges will never be achieved in a real-world environment. Nevertheless, the results of the Free Space Path Loss model are useful as a benchmark as they enable us to compare them with different frequencies and technologies. For instance, the maximum range of LoRa at $868 \mathrm{MHz}$ calculated with the FSPL model equals $921 \mathrm{~km}$, which is almost seven times the range of LoRa at $2.4 \mathrm{GHz}$.

For the indoor range estimation of $2.4 \mathrm{GHz} \mathrm{LoRa}$, an indoor path loss model was evaluated. In order to provide the highest possible accuracy, a model based on real-world measurements was adopted and slightly modified, taking into account both wall and interaction loss [28]. The estimated range varies from $18 \mathrm{~m}$ to $74 \mathrm{~m}$, depending on the SF and BW. It should be noted that a path loss exponent of 5 was chosen, simulating an obstructed indoor environment. However, in an indoor LoS scenario, the range might therefore be increased.

Finally, the maximum communication range in an urban environment was found to be $867 \mathrm{~m}$. As indicated in Figure 4, the range at an SF of 10 and a BW equal to $406 \mathrm{kHz}$ is $443 \mathrm{~m}$. This is similar to the results of the experiments with the SX1280 chipset carried out by Wolf et al. [17]. They found that ToF ranging with the aforementioned SF and BW failed for ranges over about $500 \mathrm{~m}$. Although they only investigated the ranging feature at an SF of 10 and a BW equal to $406 \mathrm{kbit} / \mathrm{s}$ and $1625 \mathrm{kbit} / \mathrm{s}$, this partially validates our range estimations of the ECC-33 path loss model. 
Besides the communication range, we investigated the data rates for all combinations of SFs and BWs and consequently associated this information with the highest achievable range. The highest possible data rate of LoRa at $2.4 \mathrm{GHz}$ equals $253.91 \mathrm{kbit} / \mathrm{s}$, which is almost seven times higher than the maximum data rate of LoRa at $868 \mathrm{MHz}$. This data rate can be achieved if the distance between the transmitter and receiver is not greater than $3739 \mathrm{~m}, 18 \mathrm{~m}$ and $3 \mathrm{~m}$ in a free space, indoor and urban environment, respectively.

Some significant differences in terms of range arise when comparing LoRa to other technologies operating in the $2.4 \mathrm{GHz}$ band. As mentioned earlier, the range of the latest Bluetooth standard equals $50 \mathrm{~m}$ and $165 \mathrm{~m}$ in an indoor and outdoor environment, respectively. Moreover, the maximum range of $2.4 \mathrm{GHz}$ Wi-Fi networks typically varies around $100 \mathrm{~m}$. Thus, the outdoor range of LoRa is almost three times larger than the outdoor range of BLE 5 and more than eight times larger compared to typical IEEE 802.11 networks. This is mainly due to the lower bandwidth and data rates used in LoRa, as well as the robustness of the LoRa-modulated signal. These numbers clearly indicate the significant difference in intended applications between LoRa (such as long-range communication and localization) and Wi-Fi and Bluetooth (such as video and audio streaming).

Since LoRa modulation at $2.4 \mathrm{GHz}$ has a higher bandwidth than LoRa modulation at $868 \mathrm{MHz}$, the rising edge of a signal pulse can be determined more accurately. Therefore, we expect that time-based localization methods for this technology will result in lower estimation errors. However, the results in this paper show that it is not possible to achieve the same long communication ranges as LoRa at $868 \mathrm{MHz}$ and with other sub-GHz LPWANs. Therefore, more LoRa receivers have to be deployed to cover wide areas, which makes it a less feasible solution to build large public networks. On the other hand, $2.4 \mathrm{GHz}$ LoRa is an interesting option for both communication and localization in privately deployed networks that are purposed for asset tracking and monitoring in large warehouses, construction sites, farms, etc.

\section{Conclusions}

With the move from sub-GHz frequency bands to the globally available $2.4 \mathrm{GHz}$ ISM band, hardware manufacturers are able to design a uniform LoRa chipset which functions independently of the region of deployment. However, as a consequence of moving to a higher frequency, the range of LoRa is reduced when compared to the sub-GHz range of several kilometers. In this paper, we first provide an overview of LoRa operating in the $2.4 \mathrm{GHz}$ band. By calculating the link budget of a chipset operating in this band, the range of a LoRa modulated signal is estimated in a free space and in indoor and outdoor scenarios. When compared to other technologies operating in the $2.4 \mathrm{GHz}$ band, LoRa outperforms them in terms of communication range, due to the configurable SF and bandwidth. Thus, when configuring a LoRa channel at $2.4 \mathrm{GHz}$, a trade-off between range and data rate should be taken into account. Moreover, this trade-off leads to more flexible applications, such as the localization of assets in a private LoRaWAN network, which will be investigated in future work.

Author Contributions: The presented work was carried out in collaboration with all of the authors. Conceptualization, M.W.; methodology, T.J. and M.W.; software, T.J. and N.B.; validation, M.A.; investigation, T.J. and N.B.; writing-original draft preparation, T.J., N.B. and M.A.; writing-review and editing, R.B. and M.W.; visualization, T.J., N.B. and R.B.; supervision, R.B. and M.W.; All authors have read and agreed to the published version of the manuscript.

Funding: Thomas Janssen is funded by the Fund For Scientific Research (FWO) Flanders under grant number 1S03821N. Part of this research has received funding from the European Union's Horizon 2020 research and innovation program under grant agreement No 769267 (PortForward project).

Conflicts of Interest: The authors declare no conflict of interest.

\section{References}

1. Sendra, S.; García, L.; Lloret, J.; Bosch, I.; Vega-Rodríguez, R. LoRaWAN Network for Fire Monitoring in Rural Environments. Electronics 2020, 9, 531. [CrossRef] 
2. Mackey, A.; Spachos, P. LoRa-based Localization System for Emergency Services in GPS-less Environments. In Proceedings of the INFOCOM 2019-IEEE Conference on Computer Communications Workshops (INFOCOM WKSHPS), Paris, France, 29 April-2 May 2019; pp. 939-944. [CrossRef]

3. Buurman, B.; Kamruzzaman, J.; Karmakar, G.; Islam, S. Low-Power Wide-Area Networks: Design Goals, Architecture, Suitability to Use Cases and Research Challenges. IEEE Access 2020, 8, 17179-17220. [CrossRef]

4. Cilfone, A.; Davoli, L. LoRaFarM: A LoRaWAN-Based Smart Farming Modular IoT Architecture. Sensors 2020. [CrossRef]

5. Semtech. Semtech SX128x Long Range Datasheet. 2019. Available online: https://semtech.my.salesforce. $\mathrm{com} / \mathrm{sfc} / \mathrm{p} / \#$ E0000000JelG/a/2R000000HVET/HfcgiChyabtiPTh6EjcDM6ZEwAOQV7IirEmRULgggMM (accessed on 26 May 2020).

6. Aernouts, M.; BniLam, N.; Podevijn, N.; Plets, D.; Joseph, W.; Berkvens, R.; Weyn, M. Combining TDoA and AoA with a Particle Filter in an Outdoor LoRaWAN Network. In Proceedings of the IEEE/ION PLANSx Position, Location and Navigation Symposium (PLANS), Portland, OR, USA, 20-23 April 2020; IEEE: St-Louis, MO, USA, 2020.

7. Augustin, A.; Yi, J.; Clausen, T.; Townsley, W. A Study of LoRa: Long Range \& Low Power Networks for the Internet of Things. Sensors 2016, 16, 1466. [CrossRef]

8. Ayoub, W.; Samhat, A.E.; Nouvel, F.; Mroue, M.; Prévotet, J.C. Internet of Mobile Things: Overview of LoRaWAN, DASH7, and NB-IoT in LPWANs standards and Supported Mobility. IEEE Commun. Surv. Tutorials 2019, 21, 1561-1581. [CrossRef]

9. Bernier, C.; Dehmas, F.; Deparis, N. Low Complexity LoRa Frame Synchronization for Ultra-Low Power Software-Defined Radios. IEEE Trans. Commun. 2020, 68, 3140-3152. [CrossRef]

10. Sisinni, E.; Ferrari, P.; Fernandes Carvalho, D.; Rinaldi, S.; Marco, P.; Flammini, A.; Depari, A. LoRaWAN Range Extender for Industrial IoT. IEEE Trans. Ind. Informatics 2020, 16, 5607-5616. [CrossRef]

11. Janssen, T.; Aernouts, M.; Berkvens, R.; Weyn, M. Outdoor Fingerprinting Localization Using Sigfox. In Proceedings of the 2018 International Conference on Indoor Positioning and Indoor Navigation (IPIN), Nantes, France, 24-27 September 2018.

12. Janssen, T.; Berkvens, R.; Weyn, M. Comparing Machine Learning Algorithms for RSS-Based Localization in LPWAN. In International Conference on P2P, Parallel, Grid, Cloud and Internet Computing (3PGCIC-2019); Barolli, L., Hellinckx, P., Natwichai, J., Eds.; Springer International Publishing: Antwerp, Belgium, 2019; pp. 726-735. [CrossRef]

13. Janssen, T.; Weyn, M.; Berkvens, R. A Primer on Real-world RSS-based Outdoor NB-IoT Localization. In Proceedings of the International Conference on Localization and GNSS (ICL-GNSS), Tampere, Finland, 2-4 June 2020.

14. BniLam, N.; Steckel, J.; Weyn, M. Synchronization of multiple independent subarray antennas: An application for angle of arrival estimation. IEEE Trans. Antennas Propag. 2019, 67, 1223-1232. [CrossRef]

15. Bnilam, N.; Joosens, D.; Steckel, J.; Weyn, M. Low Cost AoA Unit for IoT Applications. In Proceedings of the 13th European Conference on Antennas and Propagation (EuCAP 2019), Krakow, Poland, 31 March-5 April 2019.

16. Semtech. Application Note: Ranging with the SX1280 Transceiver. 2017. Available online: https://semtech. my.salesforce.com/sfc/p/\#E0000000JelG/a /44000000MDiH/OF02Lve2RzM6pUw9gNgSJXbDNaQJ_ NtQ555rLzY3UvY (accessed on 5 July 2020).

17. Wolf, F.; Le Deroff, K.; de Rivaz, S.; Deparis, N.; Dehmas, F.; Cances, J.P. Benchmarking of Narrowband LPWA Physical Layer Ranging Technologies. In Proceedings of the 2019 16th Workshop on Positioning, Navigation and Communications (WPNC), Bremen, Germany, 23-24 October 2019; pp. 1-6. [CrossRef]

18. Mahanti, A.; Carlsson, N.; Williamson, C.; Arlitt, M. Ambient interference effects in Wi-Fi networks. In International Conference on Research in Networking; Springer: Berlin, Heidelberg Germany, 2010; Volume 6091 LNCS, pp. 160-173. [CrossRef]

19. Polak, L.; Milos, J. Performance analysis of LoRa in the $2.4 \mathrm{GHz}$ ISM band: coexistence issues with Wi-Fi. Telecommun. Syst. 2020, 74, 299-309. [CrossRef]

20. Karvonen, H.; Pomalaza-Ráez, C.; Mikhaylov, K.; Hämäläinen, M.; Iinatti, J. Experimental Performance Evaluation of BLE 4 Versus BLE 5 in Indoors and Outdoors Scenarios. In Advances in Body Area Networks; Springer: Cham, Switzerland, 2019; pp. 235-251. [CrossRef]

21. Knight, M.; Seeber, B. Decoding LoRa: Realizing a Modern LPWAN with SDR. Proc. GNU Radio Conf. 2016, 1. 
22. Vangelista, L. Frequency Shift Chirp Modulation: The LoRa Modulation. IEEE Signal Process. Lett. 2017, 24, 1818-1821. [CrossRef]

23. Robyns, P.; Quax, P.; Lamotte, W.; Thenaers, W. A multi-channel software decoder for the LoRa modulation scheme. In Proceedings of the 3rd International Conference on Internet of Things, Big Data and Security (IoTBDS 2018), Funchal, Madeira, Portugal, 19-21 March 2018; pp. 41-51. [CrossRef]

24. Ghanaatian, R.; Afisiadis, O.; Cotting, M.; Burg, A. Lora Digital Receiver Analysis and Implementation. In Proceedings of the 2019 IEEE International Conference on Acoustics, Speech and Signal Processing (ICASSP), Brighton, UK, 12-17 May 2019; pp. 1498-1502. [CrossRef]

25. Popovic, B. Generalized chirp-like polyphase sequences with optimum correlation properties. IEEE Trans. Inf. Theory 1992, 38, 1406-1409. [CrossRef]

26. Hua, M.; Wang, M.; Yang, K.W.; Zou, K.J. Analysis of the frequency offset effect on Zadoff-Chu sequence timing performance. IEEE Trans. Commun. 2014, 62, 4024-4039. [CrossRef]

27. Heereman, F.; Joseph, W.; Tanghe, E.; Plets, D.; Verloock, L.; Martens, L. Path loss model and prediction of range, power and throughput for 802.11n in large conference rooms. AEU-Int. J. Electron. Commun. 2012, 66, 561-568. [CrossRef]

28. Plets, D.; Joseph, W.; Vanhecke, K.; Tanghe, E.; Martens, L. Simple Indoor Path Loss Prediction Algorithm and Validation in Living Lab Setting. Wirel. Pers. Commun. 2013, 68, 535-552. [CrossRef]

29. Razali, N.A.M.; Habaebi, M.H.; Zulkurnain, N.F.; Islam, M.R.; Zyoud, A. The distribution of path loss exponent in 3D indoor environment. Int. J. Appl. Eng. Res. 2017, 12, 7154-7161.

30. Mollel, M.; Kisangiri, M. Comparison of Empirical Propagation Path Loss Models for Mobile Communication. Comput. Eng. Intell. Syst. 2014, 5, 1-11.

31. Iskander, M.F. Channel Characterization and Propagation Models for LTE Path Loss Prediction in Urban and Suburban Ghana. Int. J. Wirel. Mob. Networks (IJWMN) 2019, 11, 23-32.

(c) 2020 by the authors. Licensee MDPI, Basel, Switzerland. This article is an open access article distributed under the terms and conditions of the Creative Commons Attribution (CC BY) license (http:/ / creativecommons.org/licenses/by/4.0/). 